\title{
Triplet-Triplet Annihilation PLGA-Nanoparticles for Cancer Bioimaging
}

Olena Vepris ${ }^{\dagger}$, Christina Eich ${ }^{\dagger}$, Yansong Feng ${ }^{\ddagger}$, Hong Zhang ${ }^{\ddagger}$, Eric. L. Kaijzel ${ }^{\dagger}$, Luis J. Cruz ${ }^{\dagger *}$ Authors' affiliations:

†Department of Radiology, Leiden University Medical Center (LUMC)

†Van ‘t Hoff Institute for Molecular Sciences. University of Amsterdam, Science Park 904, 1098 XH, Amsterdam, the Netherlands

Correspondence to:

Luis J. Cruz, Ph.D, Group leader Translational Nanobiomaterials and Imaging. Department of Radiology, Room C2-187h, Leiden University Medical Centre (LUMC), Albinusdreef 2, 2333 ZA Leiden, The Netherlands. Phone: +31715265764. Email: 1.j.cruz_ricondo@lumc.nl. 


\section{Abstract}

Triplet-triplet annihilation upconversion (TTA-UC) nanoparticles (NPs) have emerged as imaging probes and therapeutic probes in recent years due to their excellent optical properties. In contrast to lanthanide ions-doped inorganic materials, highly efficient TTA-UC can be generated by low excitation power density, which makes it suitable for clinical applications. In the present study, we used biodegradable poly(lactic-co-glycolic acid) (PLGA)-NPs as delivery vehicle for TTA-UC based on the heavy metal porphyrin Platinum(II) octaethylporphyrin (PtOEP) and the polycyclic aromatic hydrocarbon 9,10diphenylanthracene (DPA) as photosensitizer/emitter pair. TTA-UCPLGA-NPs were successfully synthesized according to an oil-in-water emulsion and solvent evaporation method. After physicochemical characterization, UCefficacy of TTA-UC-PLGA-NPs was assessed in vitro and ex vivo. TTA-UC could be detected in the tumor area 96 hours after in vivo administration of TTA-UCPLGA-NPs, confirming the integrity and suitability of PLGA-NPs as TTA-UC in vivo delivery system. Thus, this study provides proof-of-concept that the advantageous properties of PLGA can be combined with the unique optical properties of TTA-UC for the development of advanced nanocarriers for simultaneous in vivo molecular imaging and drug delivery.

Keywords: Photon upconversion, Triplettriplet annihilation, In vivo imaging, PLGA, nanoparticles 


\section{Introduction}

Since the introduction of imaging techniques to diagnosis and treatment of different pathologies, great progress has been achieved in the field of medical imaging, and the variety of imaging agents become more sophisticated in terms of efficacy, safety and target-specificity ${ }^{1}$.

In the last decade, photon upconversion (UC), a process that converts low-energy into high-energy light ${ }^{2,3}$, has become stateof-art in the field of in vitro and in vivo biomedical imaging due to several unique optical properties: i) large hypsochromic shift, ii) sharp emission peak, iii) long luminescence shelf-life and iv) high photostability ${ }^{4-6}$. Photon UC is a combination of photophysical processes and it can be achieved via different energy transfer mechanisms using different materials, such as rare-earth metals, metalloporphyrins and organic polyaromatic hydrocarbons ${ }^{2,3}$.

Triplet-triplet annihilation (TTA) is a special form of UC, resulting in superior optical properties, such as intense absorption of excitation light and high UC quantum yield, while using low excitation power density ${ }^{3,7,8}$; the latter makes TTA$\mathrm{UC}$ in particular interesting for biomedical applications. In TTA-UC, the energy transfer between a sensitizer and an emitter molecule takes place through a series of subsequent nonradiative mechanisms, such as intersystem crossing (ISC) and triplettriplet energy transfer (TTET) (Figure 1) $3,7,8$.

When electromagnetic radiation "hits" a sensitizer molecule its electrons will be promoted from the ground to a singlet excited state, and then transferred to a triplet excited state nonradiatively. The released energy that accompanies the transition of the triplet excited electron to the ground singlet state will be transferred to an emitter/acceptor molecule to pump the latter to an excited triplet state. If two of these triplet excited state molecules are close enough, the interaction between the two will cause the transition of one of them to the ground singlet state and the other to a singlet excited state, i.e. the collision results in an annihilation of the triplet excited state of one acceptor molecule and promotion of another one to the singlet excited state (Figure 1). 


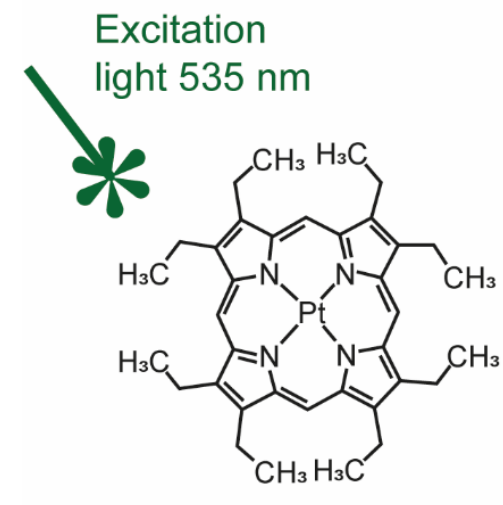

PtOEP

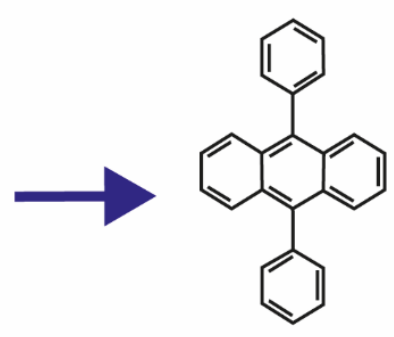

DPA

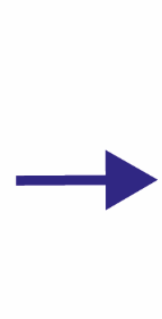<smiles>c1ccc(-c2ccccc2-c2ccccc2-c2ccccc2)cc1</smiles>

DPA

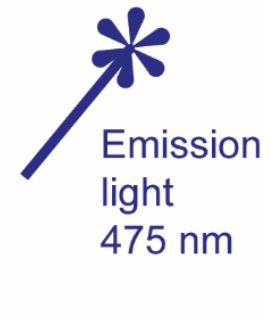

Emission $475 \mathrm{~nm}$

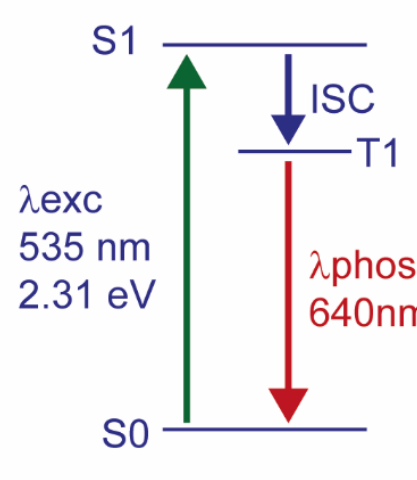

Photosensitizer
S1

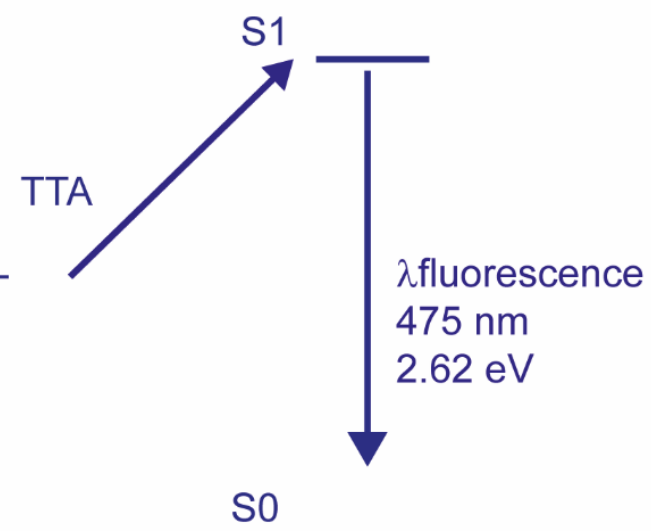

So

Emitter 1

\section{Emitter 2}

Figure 1. Jablonski diagram describing the energy levels of the electrons involved in energy transfer between PtOEP and DPA molecules. The energy values were calculated based on theoretical data.

The UC-process is a highly improbable event according to the quantum mechanical principle, since it consists of a series of spin-forbidden transitions ${ }^{9}$. It means that the UC-process should be boosted under favourable conditions that promote those transitions. For instance, to promote ISC, which is the transition of a molecule from a singlet electronic excited state to a triplet electron state, it is advantageous to choose molecules with strong spin-orbit coupling and an expanded $\pi$ conjugated system. Metalloporphyrins, such as the green absorbing platinum octaethylporphyrin (PtOEP), possess both requirements due to the presence of a transition metal that promotes a spin-forbidden transition and a cyclic $\pi$ conjugated system that enhances 
ISC yield ${ }^{10,11,12}$. More recently, lanthanide complexes of porphyrinoids inside of nanomicelles and mesoporous silica NPs were employed for in vivo imaging in HeLA cells, demonstrating the potential of porphyrinoids as sensitizers in TTA-UC ${ }^{13}$. In the present study, we describe an approach whereby TTA-UC is combined with biocompatible poly(lactic-co-glycolic acid) (PLGA) to create an UC-PLGAnanoparticle (NP) system as a potential tool for cancer visualization. NPs, such as those made of biodegradable and FDA-approved PLGA, protect their payload from premature degradation ${ }^{14-20}$, are well described, chemically adaptable and can guide their payload inside target cells in vitro and in vivo ${ }^{19-22}$.

To generate TTA-UC, we selected PtOEP as the photosensitizer and 9,10diphenylanthracene (DPA) as the annihilator; a combination that has been reported to result in highly efficient UC yield 23,24. PtOEP and DPA were successfully encapsulated into the hydrophobic core of PLGA-NPs. Phosphorescence and UC fluorescence signals resulting from TTA-UC-PLGA-NPs could be detected in vitro, in vivo and ex vivo up to 96 hours after injection. We conclude that PLGA provides a suitable environment for TTA-UC, which could be utilized for simultaneous optical imaging and drug delivery in vivo. 


\section{Results and Discussion}

\section{Preparation, physicochemical}

characterization and cytotoxicity of TTA-

\section{UC-PLGA-NPS}

In the present study, TTA-UC-PLGA-NPs were successfully synthesized applying an oil-in-water emulsion and solvent evaporation method, as previously described 19,20,22,25. Briefly, $100 \mathrm{mg}$ of PLGA and $1 \mathrm{mg}$ of each chromophore were dissolved in $3 \mathrm{ml}$ of dichloromethane (DCM). The mixture of chromophores and PLGA polymer was emulsified under sonication in the presence of PVA. After removal of the organic solvent, the TTAUC-PLGA-NPs were collected by centrifugation, washed and freeze-dried. The oil-in-water emulsion and solvent evaporation method led to an encapsulation efficiency (EE) of PtOEP and DPA molecules of $24 \%$ and $40 \%$, respectively, as determined by nanodrop measurement of dissolved NPs (data not shown).

Due to the high sensitivity of the porphyrin triplet states to oxygen quenching, the actual lifetime of the triplet state depends on the solvent, its purity and on its oxygen content ${ }^{26}$. To protect the optical properties of chromophores, PtOEP and DPA were dissolved in DCM. To ensure that encapsulation into PLGA-NPs did not affect the optical properties of PtOEP and DPA, TTA-UC-PLGA-NPs were dissolved in dimethylformamide (DMF) and analysed by absorption curve analysis and compared to pure compounds (Figure 2). The absorption spectrum of PtOEP depicts the characteristic shape of porphyrin absorption: a strong absorption peak at $380 \mathrm{~nm}$ (Soret band) was accompanied by a weaker absorption peak located at 501 and $533 \mathrm{~nm}$ (Q-band) ${ }^{27}$ (Figure 2A). DPA, the emitter for the TTA-UC system, showed several absorption peaks between $350 \mathrm{~nm}$ and $400 \mathrm{~nm}$ (Figure 2B). When the TTAUC-PLGA-NPs were dissolved in DMF, the absorption spectrum showed the combined characteristic optical profiles of PtOEP and DPA (Figure 2C). In conclusion, absorption spectrum analysis of TTA-UC-PLGA-NPs confirmed the successful encapsulation of both chromophores into the PLGA polymer, while the optical properties of PtOEP and DPA were maintained. 
Vepris et al, Figure 2
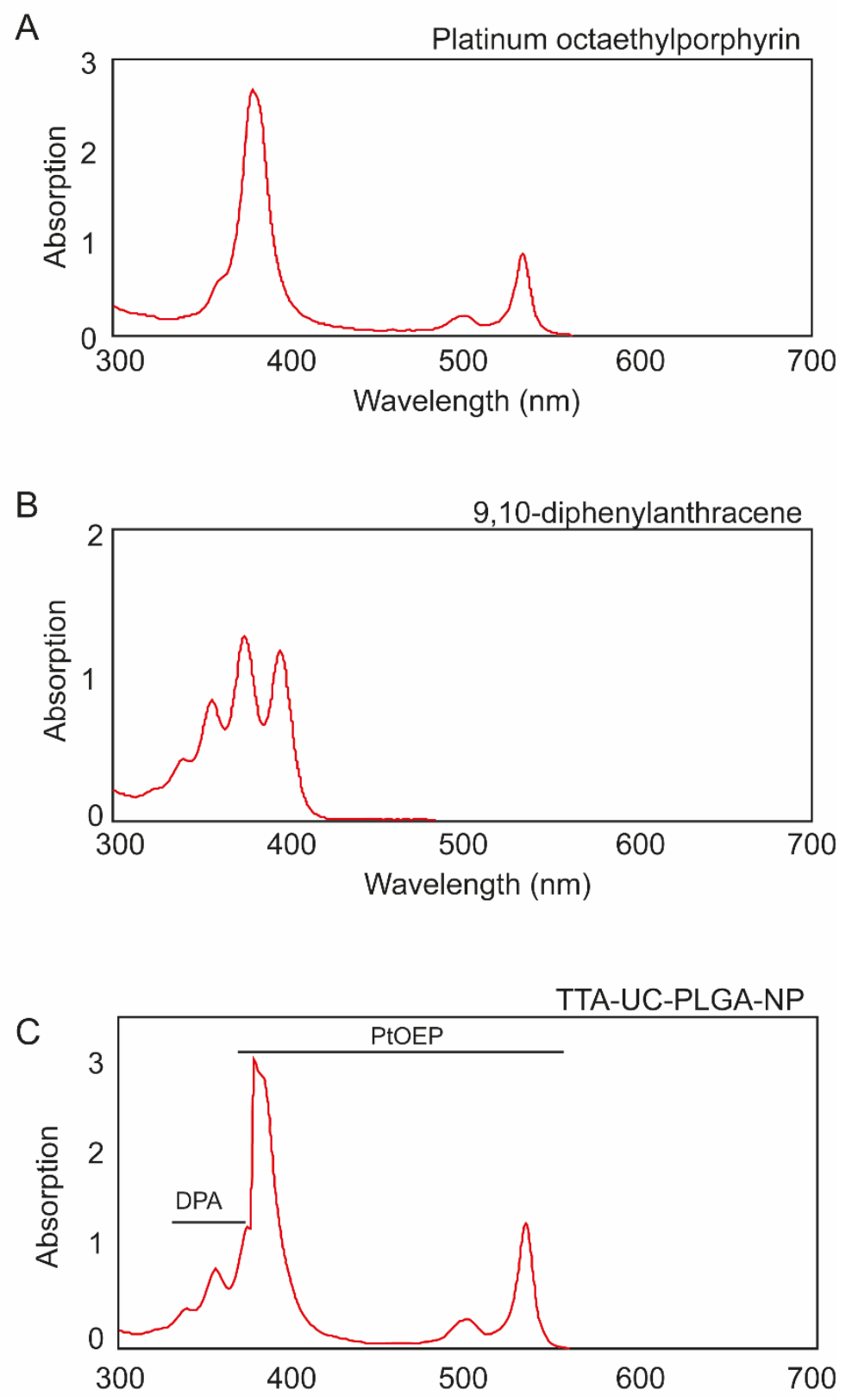

Figure 2. Individual absorption spectra of (A) platinum octaethylporphyrin (PtOEP) and (B) 9,8-diphenylanthracene (DPA) each dissolved in dimethylformamide (DMF). (C) TTA-UCPLGA-NPs were hydrolysed overnight in $0.8 \mathrm{M} \mathrm{NaOH}$ at $37^{\circ} \mathrm{C}$ and the absorption spectrum of co-encapsulated PtOEP and DPA were measured. 


\begin{tabular}{cccccc}
\hline NPs & Size $(\mathbf{n m})$ & $\begin{array}{c}\text { Zeta } \\
\text { potential } \\
(\mathbf{m V})\end{array}$ & PDI & $\begin{array}{c}\text { Loading } \\
(\boldsymbol{\mu g} / \mathbf{m g})\end{array}$ & $\begin{array}{c}\text { Encapsulation } \\
\text { efficiency }(\%)\end{array}$ \\
\hline $\begin{array}{c}\text { TTA-UC } \\
\text { PLGA }\end{array}$ & $197.8 \pm 47.5$ & $-30.7 \pm 5.0$ & 0.4 & $\begin{array}{c}\text { PtOEP: } 244.0 \\
\text { DPA: } 396.0\end{array}$ & $\begin{array}{c}\text { PtOEP: } 24.4 \\
\text { DPA: } 39.6\end{array}$ \\
\hline
\end{tabular}

Table 1. Physicochemical properties of NPs. Data are present as average \pm SD $(\mathrm{N}=3)$

The size of the NPs was determined by dynamic light scattering (DLS) analysis as $\sim 200 \mathrm{~nm}$ in diameter (Table 1, Figure 3A) and the polydispersity index (PDI) value of
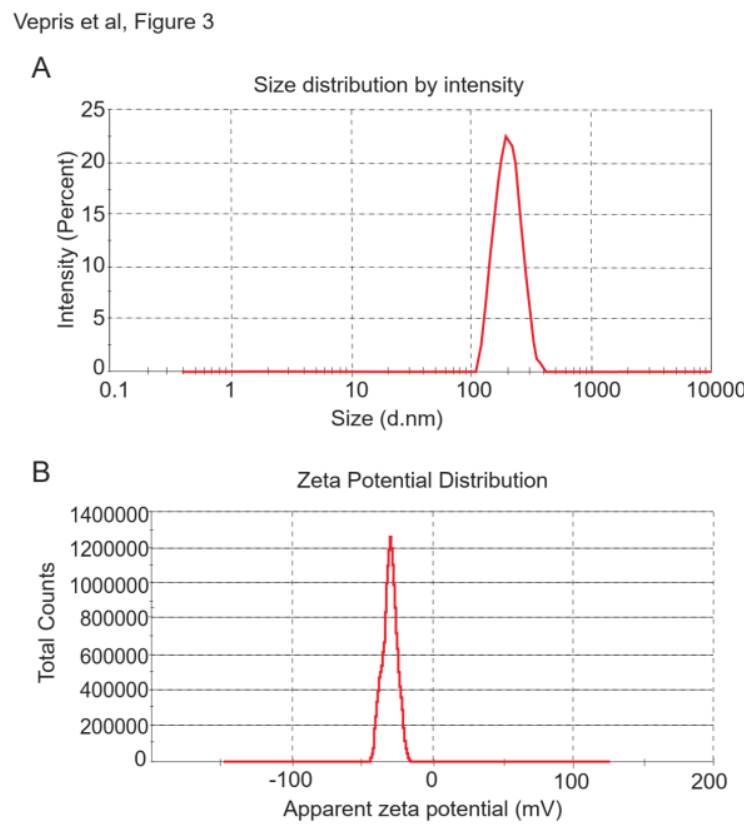

C

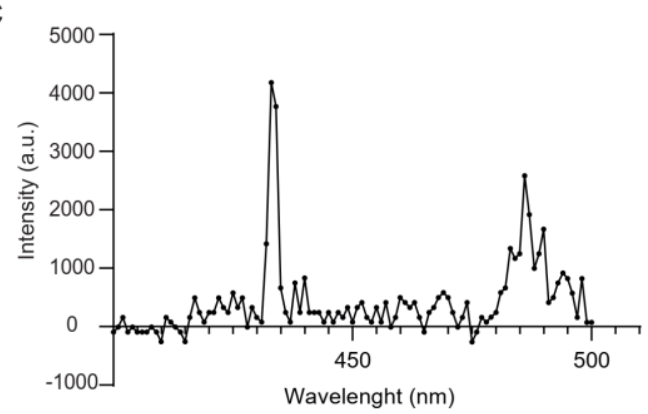

0.4 obtained from the DLS measurement indicated a homogeneous size distribution (Table 1). 
Figure 3. Physicochemical characterization of TTA-UC-PLGA-NPs. Representative (A) dynamic light scattering and (B) zeta-potential measurement of TTA-UC-PLGA-NPs reconstituted in water after freeze-drying. The average size of the nanoparticles was $198 \mathrm{~nm}$ in diameter and the average zeta potential value was $-31 \mathrm{mV}$. (C) TTA-UC-PLGA-NPs were dissolved in water and directly analysed. The NPs in solution were excited at $535 \mathrm{~nm}$ and the emission was collected using a photofluorometer.

NPs smaller than $10 \mathrm{~nm}$ are known to be eliminated by renal excretion ${ }^{28}$, while NPs with sizes ranging from 50 to $300 \mathrm{~nm}$ showed extended circulation times in the blood stream compared to NPs of larger size $^{29}$. Hence, TTA-UC-PLGA-NPs of 200 $\mathrm{nm}$ have the ideal size for prolonged systemic circulation times; an important prerequisite to reach the tumor site. In addition, ZetaSizer measurement showed that the surface charge of the NPs was on average $-30 \mathrm{mV}$ (Table 1, Figure 3B). The surface charge is a crucial factor to determine the stability of the NPs in solution, but also affects cellular uptake, biodistribution and cytotoxicity. A high surface charge guarantees an electrostatic stabilization of the NPs due to a strong surface repulsion between NPs of the same charge. According to the measured value, it is possible to assume that the NPs are stable in suspension for a prolonged period of time 30,31 .

Next, we investigated the optical properties of the TTA-UC system after encapsulation into PLGA-NPs. To this purpose, TTA-UCPLGA-NPs were dissolved in water and excited at 535nm (Figure 3C). The TTAUC signal could be detected at $433 \mathrm{~nm}$, confirming the integrity of the TTA-UC system after encapsulation into PLGA.

The charge of UC-NPs has been shown to affect the intracellular localization and cellular cytotoxicity ${ }^{32}$. Positively charged UC-NPs localized to mitochondria, while negatively charged UC-NPs preferentially localized to lysosomes and the cytoplasm, which was associated with lower cellular cytotoxicity ${ }^{32}$. To assess potential toxicity of our NPs, TTA-UC-PLGA-NPs were incubated at different concentrations (25$200 \mu \mathrm{g} / \mathrm{ml}$ ) for 72 hours with OVCAR-3 ovarian cancer cells, and the cellular toxicity was determined by MTS assay (Figure 4). TTA-UC-PLGA-NPs were compared to empty control PLGA-NPs. No toxicity of TTA-UC-PLGA-NPs or empty PLGA-NPs on OVCAR-3 cells was observed after 72 hours (Figure 4). 
Vepris et al, Figure 4

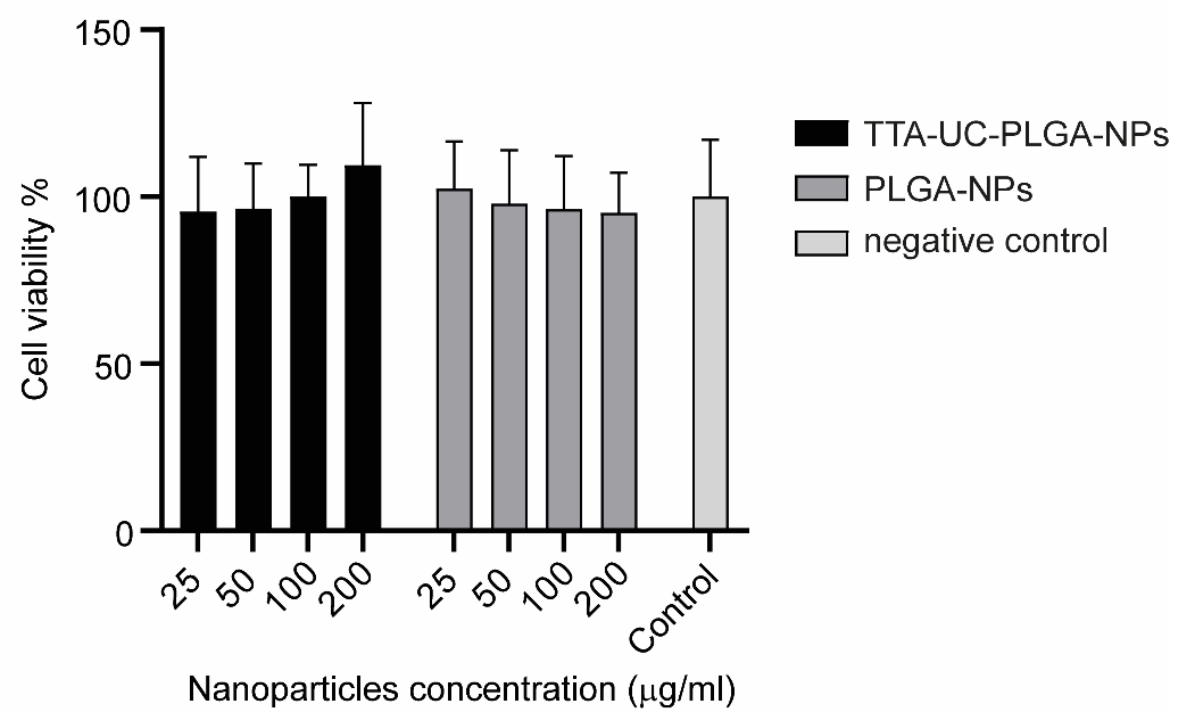

Figure 4. Assessment of cellular cytotoxicity of TTA-UC-PLGA-NPs. OVCAR-3 cells were incubated with 25, 50, 100 and $200 \mu \mathrm{g} / \mathrm{ml}$ TTA-UC-PLGA-NPs or control PLGA-NPs for 72 hours. Untreated cells were used as negative control. The cell viability was assessed after 72 hours by MTS assay.

Cellular Uptake of TTA-UC-PLGA-NPs characterized by fluorescence microscopy

To assess the cellular uptake of TTA-UCPLGA-NPs, OVCAR-3 cells were incubated for 2 hours with $200 \mu \mathrm{g} / \mathrm{ml}$ TTAUC-PLGA-NPs and analysed by fluorescent microscopy. In order to confirm the intracellular localization of the NPs, the cells were counterstained with DAPI $(\lambda$ excitation $=340-380 \mathrm{~nm}, \lambda$ emission $=$ $425 \mathrm{~nm}$ ) and DiD ( $\lambda$ excitation $=676-688$ $\mathrm{nm}, \lambda$ emission $=700-742 \mathrm{~nm}$ ) for nucleus and membrane detection, respectively. To detect the TTA-UC-PLGA-NPs, the samples were excited at $542-582 \mathrm{~nm}$, and the phosphorescence signal was collected at 604-644 nm (Figure 5). TTA-UC-PLGANPs were successfully taken up by OVCAR-3 cells. The overlay of the membrane staining with the phosphorescence signal of the TTA-UCPLGA-NPs confirmed the intracellular localization of the NPs (Figure 5). 

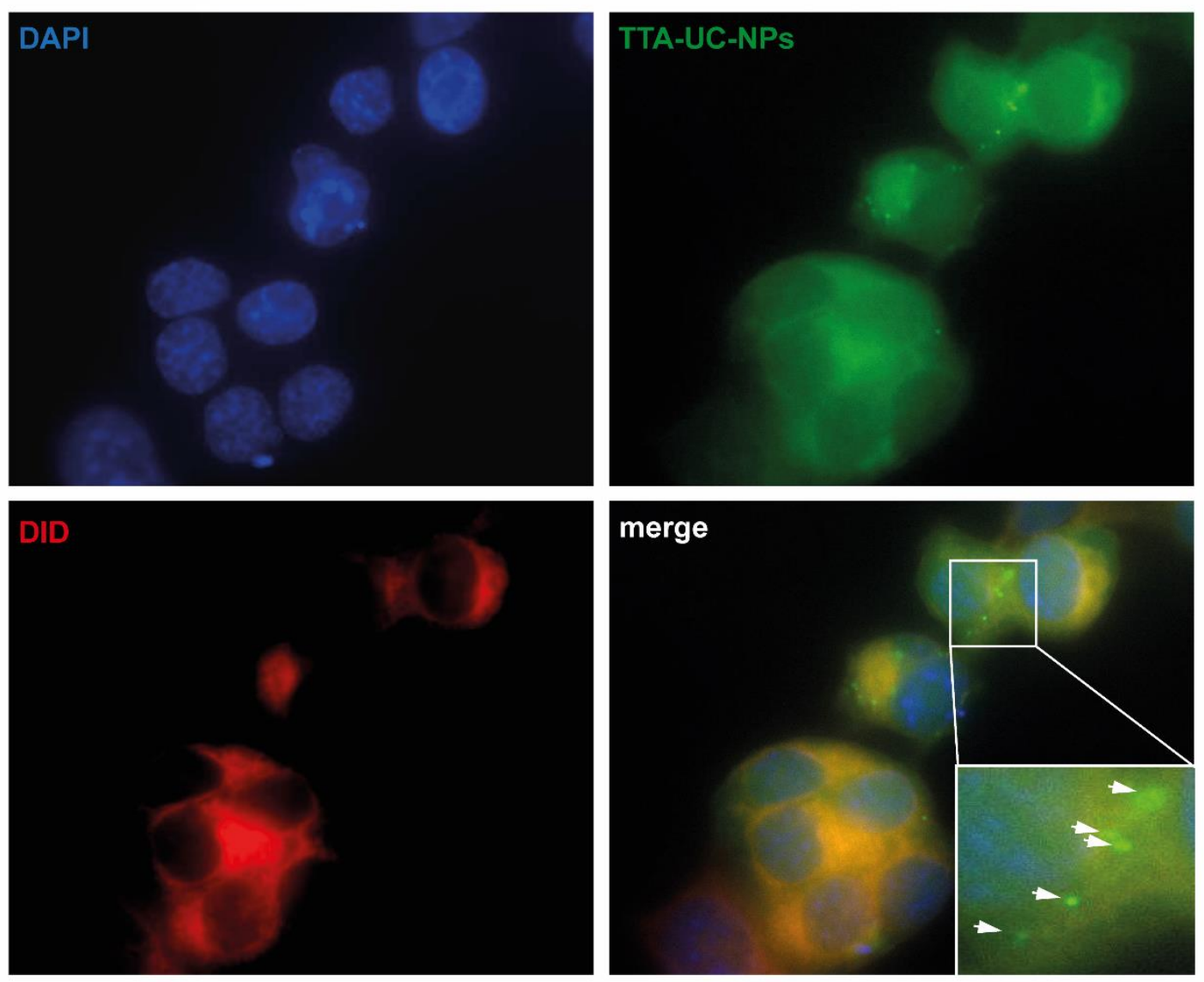

$20 \mu \mathrm{m}$

Figure 5. Cellular uptake of TTA-UC-PLGA-NPs. OVCAR-3 cells were incubated with 200 $\mu \mathrm{g} / \mathrm{ml}$ TTA-UC-PLGA-NPs for 2 hours at $37^{\circ} \mathrm{C}$ and analysed by fluorescence microscopy. The cells were fixed, and the nucleus was stained with DAPI (blue) and the cell membrane was stained with DiD (red). The phosphorescence signal (green) of the TTA-UC-PLGA-NPs was collected. The following filter cubes were used to discriminate DAPI, DiD and TTA-UCPLGA-NP-signals: DAPI, $\lambda$ exc $=340-380 \mathrm{~nm}$ and $\lambda$ emission $=425 \mathrm{~nm})$; $\operatorname{DiD}(\lambda \operatorname{exc}=676-688$ $\mathrm{nm}, \lambda$ emission $=700-742 \mathrm{~nm}$; TTA-UC-PLGA-NPs, $\lambda$ exc $=542-582 \mathrm{~nm}$, $\lambda$ emission 604-644 $\mathrm{nm}$. Scale bar $=20 \mu \mathrm{m}$.

\section{Characterization of TTA-UC-PLGA-NPS}

\section{UC properties}

The ability of TTA-UC-PLGA-NPs to generate the UC process was assessed by confocal microscopy (Figure 6). The NPs were excited at a wavelength of $535 \mathrm{~nm}$, and the emission signals were collected between $430-475 \mathrm{~nm}$ and $650-670 \mathrm{~nm}$. In our study, the emission shift towards shorter wavelengths (hypsochromic shift) went from green $(535 \mathrm{~nm})$ to blue (430-475 $\mathrm{nm}$ ) (Figure 6). It means that the energy associated with the emission wavelength was higher than the energy associated with 
the excitation wavelength, as a consequence of the anti-Stokes shift in the TTA-UC process ${ }^{33}$. On the contrary, the change in emission spectrum towards longer wavelengths (bathochromic shift), in our case $650-670 \mathrm{~nm}$, is a characteristic of all conventional luminescence processes (Figure 6). Using confocal microscopy, we could image single TTA-UC-PLGA-NPs that created dual emission wavelengths: the red emission from the phosphorescence of PtOEP and the blue emission as a result of the TTA-UC process. Thus, we confirmed the preparation of functional TTA-UCPLGA-NPs.

The dual colour feature of TTA-UC-PLGANPs was useful to improve signal-to-noise ratio and to estimate the ratio of
UC/phosphorescence signal. The overall efficiency of the TTA-UC process depends on each elementary step (ISC, TTET, TTA), on the concentration of emitter molecules and the presence of other competing molecules, such as elementary oxygen ${ }^{7,26}$. Additional challenges are encountered with when TTA-UC is integrated in a NP-system. Aggregation of photosensitizers and emitters can have detrimental effects on the optical properties of TTA-UC-NPs and, as a consequence, the loss of mobility of photosensitizers/emitters can limit the UC-process ${ }^{7,34}$. Thus, creating a highly efficient UC-NP-system without any secondary decay remains an enormous challenge for practical application ${ }^{35}$. 


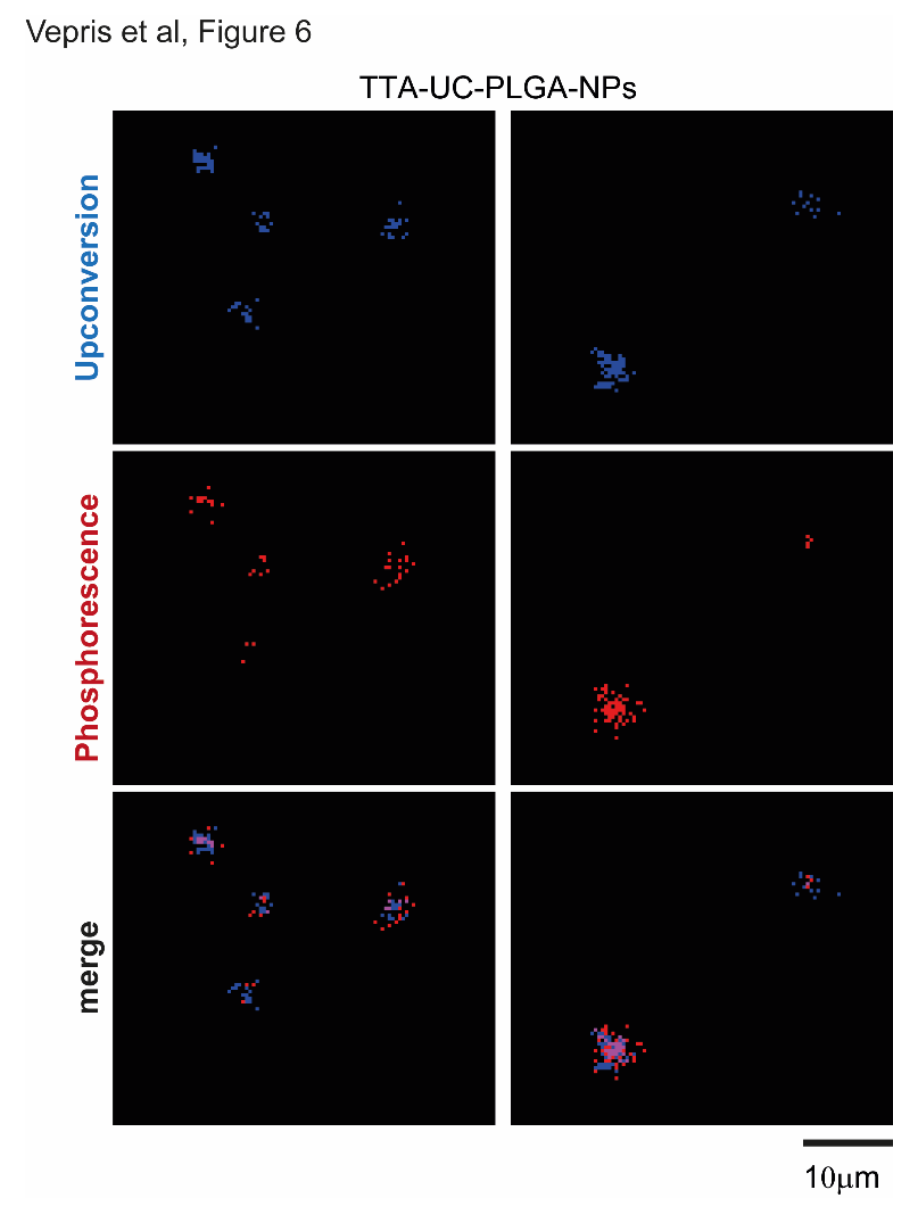

Figure 6. TTA-UC imaged by confocal microscopy. UC-TTA-PLGA-NPs were dissolved in water and imaged on a glass cover slip. The NPs were excited at $535 \mathrm{~nm}$ and the following emission wavelengths were collected: 430-475 nm (blue) = upconversion, 650-670 nm (red)= phosphorescence. Scale bar $=10 \mu \mathrm{m}$.

Stability assessment of TTA-UC-PLGANPs in solution

As PLGA-polymers are subject to hydrolysis in a water environment ${ }^{36}$ we investigated the stability of the optical properties of TTA-UC-PLGA-NPs over time (Figure 7). To this purpose, TTA-UCPLGA-NPs were dissolved in PBS and incubated at $37^{\circ} \mathrm{C}$ in shaking mode. At time point 0 , after 24 and 72 hours, the dispersion of TTA-UC-PLGA-NPs was excited at $535 \mathrm{~nm}$ and the TTA-UC and phosphorescence processes were measured at 433 and at $642 \mathrm{~nm}$, respectively (Figure 7). The strongest phosphorescence and TTA-UC signals were detected at time point 0 (0 hours post dissolution) (Figure 7A-B). After 24 hours, the phosphorescence and TTA-UC signals decreased, but could still be detected after 72 hours incubation at $37^{\circ} \mathrm{C}$. Thus, the data demonstrates that TTA-UC-PLGA-NPs are 
suitable for imaging processes lasting for at least 72 hours. The initial decrease in TTA$\mathrm{UC} /$ phosphorescence intensity within the first 24 hours is likely the result of an early hydrolysis of the PLGA polymer, resulting in the partial leaking of the chromophores out of the PLGA-core. Interestingly, while the phosphorescence signal decreased 19fold in the first 24 hours (determined at 640 $\mathrm{nm})$, the TTA-UC signal only decreased 2fold after 24 hours and 2.1-fold after 72 hours (determined at $433 \mathrm{~nm}$ ). (Figure 7AB). While both TTA-UC and phosphorescence intensities decreased over time, the TTA-UC signal relative to the Vepris et al, Figure 7

A

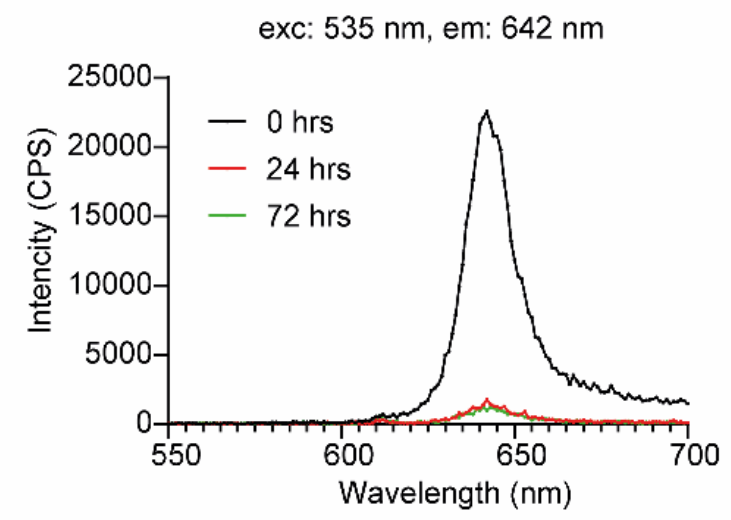

phosphorescence signal increased, indicating that the TTA-UC process became more efficient. This might indicate that the initial hydrolysis of the PLGA polymer created a favourable environment for TTA-UC. The decrease in concentration of triplet excited emitter molecules due to degradation might have been counterbalanced by an increase in photosensitizer/emitter diffusion. Diffusion of sensitizer and emitter molecules is crucial to increase the probability of intermolecular TTET and TTA to take place $^{26,34}$.

\section{$B$}

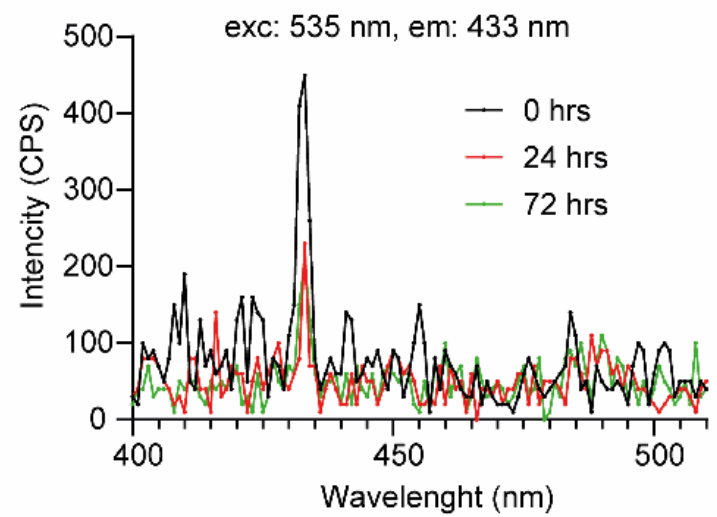

Figure 7. Stability assessment of TTA-UC-PLGA-NPs in solution. TTA-UC-PLGA-NPs were dissolved in water and incubated at $37^{\circ} \mathrm{C}$ for 72 hours. At time point 0,24 and 72 hours, samples were analysed. The NPs in solution were excited at $535 \mathrm{~nm}$ and the emission was collected using a photofluorometer. (A) Phosphorescence and (B) TTA-UC emission graphs of TTA-UC-PLGA-NPs at various time points. 
In vivo monitoring of TTA-UC-PLGANPS

Real-time imaging of FVB mice was carried out with the goal to trace TTA-UCPLGA-NPs in cancer cells in vivo. To this end, the IVIS imaging system was employed, which allowed measurement of the bathochromic emission captured at 640 $\mathrm{nm}$ (but not the UC process).

FVB mice were inoculated in the back with $1 \times 10 \mathrm{e}^{6}$ human breast adenocarcinoma cells (MCF). When the tumors reached a volume of approximately $125 \mathrm{~mm}^{3}$, TTA-UCPLGA-NPs were injected intravenously in the tail vein. At different time points post injection (3, 24, 48, 72 and 96 hours), the mice were imaged. In vivo imaging data showed that already 3 hours post injection, TTA-UC-PLGA-NPs accumulated in the tumor area (Figure 8A). The mice were monitored up to 96 hours post injection and the phosphorescence signal persisted during all imaged time points (Figure 8A-
B). Quantification of the signal showed a significant increase in phosphorescence signal in the tumor area in the first 48 hours (p 0.026) and after 96 hours (p 0.0022), indicating that more TTA-UC-PLGA-NPs accumulated at the tumor site over time. Moreover, the data showed that once accumulated in the tumor, the NPs remained at the tumor site. Since the NPs did not present any specific targeting moiety for breast adenocarcinoma cancer cells, TTA-UC-PLGA-NPs most likely accumulated at the tumor site via the enhanced permeability and retention (EPR) effect. Tumor blood vessels are naturally leaky, with large openings up to $1.5 \mathrm{~mm}$, thereby promoting the passive accumulation of NPs in tumors ${ }^{37} 38$. 
A
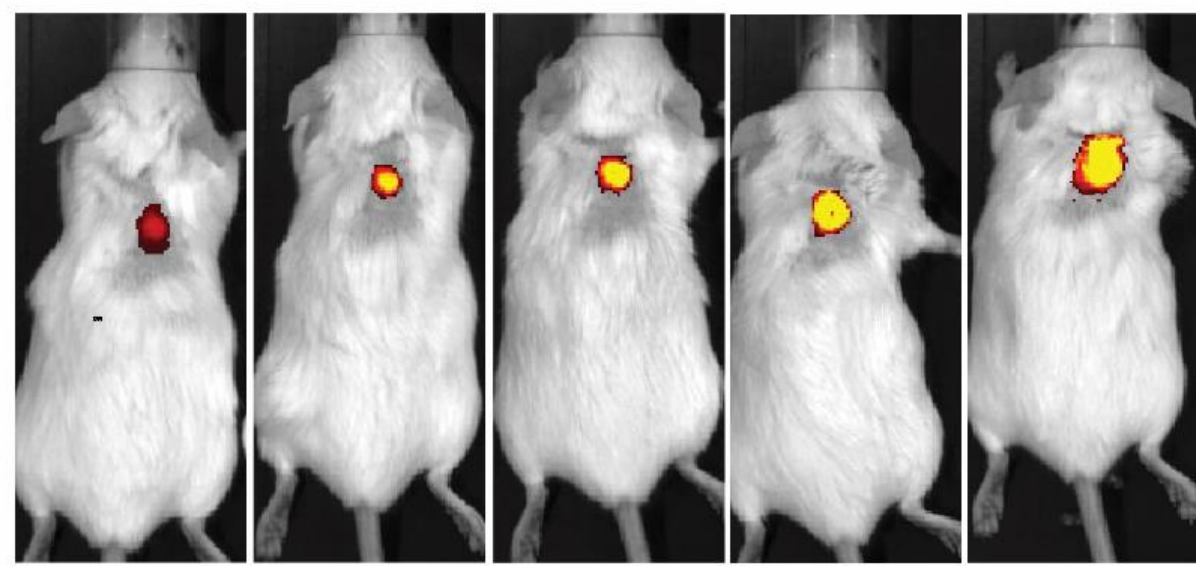

$3 \mathrm{hrs}$

24 hrs

$48 \mathrm{hrs}$

$72 \mathrm{hrs}$

$96 \mathrm{hrs}$

B

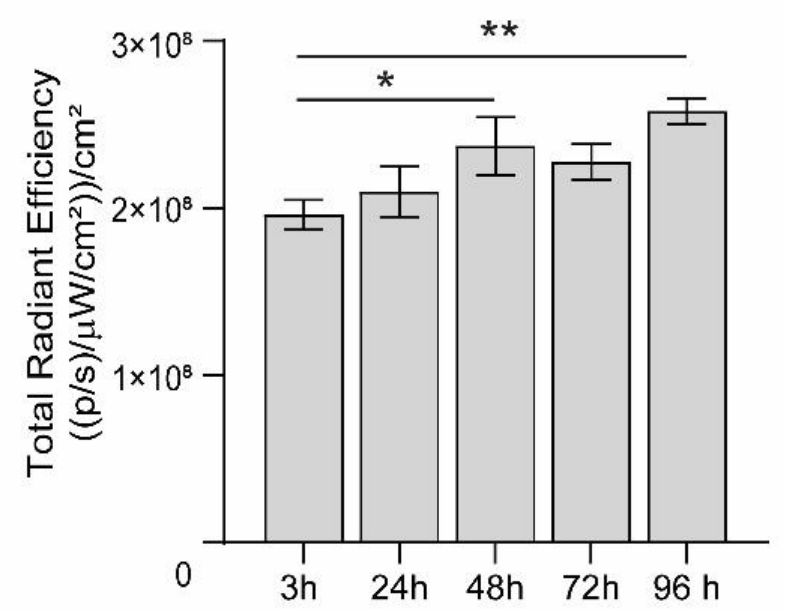

Figure 8. In vivo imaging of TTA-UC-PLGA-NPs. (a) FVB mice were inoculated in the back with $1 \times 10 \mathrm{e}^{6}$ MCF-7 cells. When the tumors reached a size of $\sim 125 \mathrm{~mm}^{3}$, mice were injected intravenously in the tail vein with $0.5 \mathrm{mg}$ of TTA-UC-PLGA-NPs and imaged 3, 24, 48, 72 and 98 hours post injection using the IVIS imaging system. (A) TTA-UC-PLGA-NPs were excited at a wavelength of $535 \mathrm{~nm}$ and the emission (phosphorescence) was collected at $640 \mathrm{~nm}$. (B) The total radiance efficiencies were calculated in the tumor areas and the values were compared. Statistical analysis was performed using Mann-Whitney test, $*=p 0.026, * *=p$ 0.0022 (6 mice per group were used). 
Measurement of TTA-UC process ex vivo 96 hours post TTA-UC-PLGA-NPs injection, breast adenocarcinoma tumors were excised and the presence of TTA-UC phenomenon was analysed ex vivo. To this end, isolated tumors were further processed for cryosectioning and imaged using a confocal microscope. The sections were excited at $514 \mathrm{~nm}$ and emissions were collected between 430-475 $\mathrm{nm}$ (to observe TTA-UC) and 650-670 nm (to observe phosphorescence) (Figure 9). Strikingly, TTA-UC and phosphorescence signals could be detected in the tumor margins and vessel-like structures 96 hours post injection of TTA-UC-PLGA-NPs. The staining pattern suggests that TTA-UCPLGA-NPs diffused into the tumor via the margins and entered into deeper tumor areas via leakages in the tumor vasculature.
The data further confirmed that, despite initial hydrolysis of the PLGA copolymers (Figure 7), the integrity of TTA-UCPLGA-NPs was maintained for at least 96 hours in vivo. 

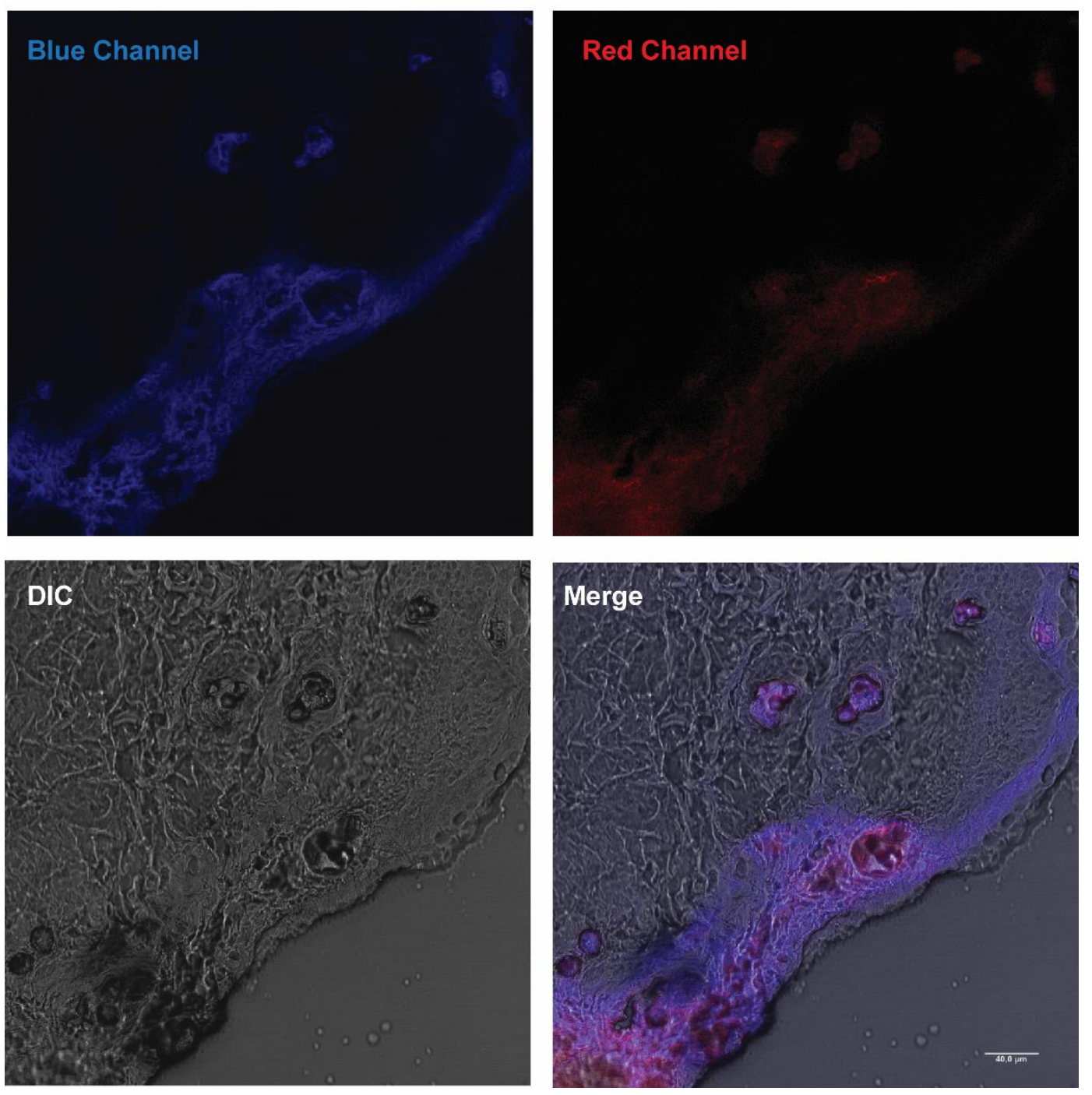

Figure 9. Ex vivo imaging of TTA-UC-PLGA-NPs. MCF-tumors were excised 96 hours post injection of TTA-UC-PLGA-NPs and tumor cryosections were imaged by confocal microscopy. All cryosections were excited at $514 \mathrm{~nm}$ and the upconversion signal (blue channel) was collected between 430-475 $\mathrm{nm}$ and the phosphorescence signal (red channel) between $650-670 \mathrm{~nm}$. Scale bar $=40 \mu \mathrm{m}$.

In conclusion, in the present study we developed a TTA-UC-PLGA-NP system by incorporating the polycyclic aromatic hydrocarbon DPA and the metalporphyrin PtOEP into a core of hydrophobic PLGAcopolymers. In vitro and ex vivo studies confirmed the presence of TTA-UC phenomenon resulting from TTA-UCPLGA-NPs. TTA-UC could be detected 96 hours post injection of TTA-UC-PLGANPs in the tumor area in vivo, confirming the integrity and suitability of PLGA-NPs as in vivo delivery system for TTA-UC, while maintaining the optical integrity of 
the TTA-UC process. This study provides proof-of-concept evidence on the feasibility of combining the advantageous properties of PLGA for drug delivery with state-of-art TTA-UC for simultaneous optical in vivo imaging. The here presented methodology could form the basis for novel TTA-UC systems encapsulated into PLGA, which can be excited in the NIR-I or NIR-II range for clinical translation. 


\section{Experimental Procedures}

\section{Materials}

PtOEP, DPA, DCM and DMF were purchased from Sigma-Aldrich $\AA$, The Netherlands. PLGA 50:50 (PLA/PGA) and water-soluble surfactant, polyvinyl alcohol (PVA), were purchased from Evonik industries AG, Germany. MTS (3-(4,5dimethylthiazol-2-yl)-5-(3-

carboxymethoxyphenyl)-2-(4-

sulfophenyl)-2H-tetrazolium, inner salt) reagent was purchased from Promega, The Netherlands. Histomount ${ }^{\mathrm{TM}}$, agar scientific, United Kingdom. All aqueous solutions were prepared with Milli-Q water.

\section{Synthesis of TTA-UC PLGA-NPs loaded} with metalloporphyrins and polycyclic aromatic hydrocarbons

The TTA-UC-PLGA-NPs were synthesized according to an oil-in-water emulsion and a solvent evaporation method. Briefly, 100 $\mathrm{mg}$ of PLGA and $1 \mathrm{mg}$ of each chromophore were dissolved in $3 \mathrm{ml}$ of DCM. The mixture of chromophores and PLGA polymer was emulsified under sonication (ultrasound tip Branson, Sonifier 250 ), with $25 \mathrm{ml}$ of water containing 500 mg of PVA for $120 \mathrm{~s}$. The newly formed emulsion was left overnight in stirring at $4^{\circ} \mathrm{C}$ in order to remove the organic solvent. The PLGA-NPs were collected by centrifugation at $14800 \mathrm{rpm}$ for $20 \mathrm{~min}$, washed three times and freeze-dried. The NPs were stored at $4{ }^{\circ} \mathrm{C}$ and rehydrated prior to use.

\section{Evaluation of physicochemical properties} of the TTA-UC-PLGA-NPS

Z-average size, PDI and zeta potential of TTA-UC-PLGA-NPs were measured using a Malvern ZetaSizer 2000 (software: ZetaSizer 7.03). Fixed scattering angle of $90^{\circ}$ at $633 \mathrm{~nm}$ was set up for the analysis. The measurements were performed after reconstitution of freeze-dried NPs in MilliQ water.

\section{Quantification PtOEP and DPA encapsulation efficiency}

The encapsulation efficiency of chromophores was determined by UV/VIS spectrophotometry using an Ultrospec 2100 pro by Amersham Bioscience. Biodegradable PLGA-NPs were hydrolysed overnight with $0,8 \mathrm{M} \mathrm{NaOH}$ at $37^{\circ} \mathrm{C}$. Concentration of dyes was calculated using a calibration curve built with known amounts of PtOEP and DPA.

\section{Cell culture}

The human ovarian carcinoma cell line OVCAR-3 and the breast cancer cell line MCF-7 were cultured in RPMI (Roswell Park Memorial Institute 1640 Medium) medium supplemented with $10 \%$ of fetal calf serum. The cells were left to attach to 
the bottom of the plate for 4 hours before treatment with NPs. After 4 hours, the NPs were added to the cells at various concentrations of 25, 50, 100, and 200 $\mu \mathrm{g} / \mathrm{ml}$. DMSO at $25 \%$ was used as a positive control. The cells treated with NPs were incubated at $37^{\circ} \mathrm{C}$ under $5 \% \mathrm{CO}_{2}$ for 72 hours. After 72 hours the medium was changed and $20 \mu \mathrm{l}$ of MTS reagent was added to each well. The ELISA reader (Molecular Devices VERSAmax Tunable Microplate Reader, Software: SoftMax Pro v5.4.1) was used to measure the absorbance value of MTS product at $490 \mathrm{~nm}$ when the colour of the medium changed from yellow to light brown. The following formula was applied to calculate the viability of cells growth: cell viability $(\%)=($ mean of absorbance value of treated sample/ mean of absorbance of negative (live) control) $\times$ 100.

\section{In vitro uptake of TTA-UC-PLGA-NPs by} OVCAR-3 cells

Fluorescence imaging of fixed OVCAR-3 cells incubated with TTA-UC-PLGA-NPs was performed with a Leica DMRA microscope using a PL APO $63 \times / 1.32-0.6$ oil objective. DAPI and Cy5 filters were used for nucleus and membrane detection, respectively, using the following protocol: Adherent cells were detached with $0.2 \%$ trypsin in PBS (Gibco by Life Technologies, The Netherlands) and seeded in 8- well chamber slides (BD Biosciences, The Netherlands) at a concentration of $2 \times 10^{4}$ cells/well. After 4 hours, the TTAUC-PLGA-NPs were added at a concentration of $200 \mu \mathrm{g} / \mathrm{ml}$ for 2 hours at $37^{\circ} \mathrm{C}$ under $5 \%$ of $\mathrm{CO}_{2}$. At the end of the incubation time, the cells were washed with PBS, fixed in $4 \%$ of paraformaldehyde (PFA) and stained with DiD labelling solution according to manufacturer protocol (Invitrogen, The Netherlands). The staining was completed adding Vectashield mounting medium containing DAPI.

\section{Measurement of $U C$ process}

Confocal microscopy imaging of TTA-UCPLGA-NPs was performed by Leica SP8 X WLL (White Light Laser) laser scanning microscope. To this end, TTA-UC-PLGANPs were dissolved at $5 \mu \mathrm{g} / \mu \mathrm{l}$ in distilled water and dropped onto a glass slide and imaged. Emissions were collected between 650-670 $\mathrm{nm}$ and $430-475 \mathrm{~nm}$; the excitation was provided at $535 \mathrm{~nm}$.

\section{Stability measurement of TTA-UC-PLGA-}

\section{NPs in solution}

In order to evaluate the stability of the TTAUC system inside the PLGA-core, the TTAUC-PLGA-NPs were dissolved in PBS and left for 0,24 and 72 hours shaking (300 rpm) at $37^{\circ} \mathrm{C}$. At each time point phosphorescence and UC-luminescence 
were measured using a spectrofluorometer (Fluorolog). The suspension of TTA-UCPLGA-NPs was excited at $535 \mathrm{~nm}$ and the emission signals were captured at 433 and $641 \mathrm{~nm}$.

\section{Animals}

Female FVB mice were purchased from Charles River Laboratories (L'Arbresle Cedex, France). Animals were housed at $22^{\circ} \mathrm{C}$ and $50 \%$ of humidity with free access to food and water, and maintained under standard 12 hours light/12 hours dark cycles.

All animal experiments were assessed according to the ethics of animal research and approved by the Animal Welfare Committee of Leiden University Medical Center, the Netherlands. All mice received humane care and were kept in compliance with the Code of Practice Use of Laboratory Animals in Cancer Research (Inspectie W\&V, July 1999). All analytical procedures were performed under isoflurane gas anaesthesia (3\% induction, $1.5-2 \%$ maintenance) in $70 \%$ pressurized air and $30 \% \mathrm{O}_{2}$, unless stated differently.

\section{In vivo monitoring of TTA-UC-PLGA-} NPS

In vivo imaging was performed using the IVIS spectrum Preclinical Imaging System (Caliper LS, Hopkinton). The images were analysed with Living Image 4.3.0 software.
First, the tumor was induced by injecting $1 \times 10^{6}$ MCF cells in $100 \mu 1$ PBS subcutaneously into the back of the mice. When the tumors reached the volume of approximately $125 \mathrm{~mm}^{3}, 0.5 \mathrm{mg}$ TTA-UCPLGA-NPs in $100 \mu \mathrm{l}$ PBS were injected into the tail vein of each mouse. At 3, 24, 72 and 96 hours post injection optical imaging of treated mice was performed. Biodistribution kinetics at each time point were measured by quantifying the fluorescence intensity in pre-set regions of interest (ROI) at the tumor site expressed as the average radiant efficiency in $\left(\mathrm{p} / \mathrm{sec} / \mathrm{cm}^{2} / \mathrm{sr} /\right) /\left(\mu \mathrm{W} / \mathrm{cm}^{2}\right)$.

\section{Measurement of TTA-UC ex vivo}

After 96 hours, the mice were sacrificed and subcutaneous tumors were surgically removed. Freshly isolated tumors were placed in tissue moulds and covered with Tissue-Tek $^{\circledR}$ O.C.T. ${ }^{\text {TM }}$ (Sakura). For snap freezing, the tumors were left on dry ice for some minutes and then stored at $-80^{\circ} \mathrm{C}$. Cryosectioning of tumors was performed using CryoStar ${ }^{\mathrm{TM}} \mathrm{NX70}$ at the working temperature between -25 and $-30^{\circ} \mathrm{C}$, the thickness of the sections was fixed between 5 and $14 \mu \mathrm{m}$. Before microscopic analysis of the tumors, the sections were washed in Milli-Q water, dried under the hood and mounted in medium containing Mowiol (Sigma) and $2.5 \%$ DABCO (Sigma). 


\section{Statistical data analysis}

Graph Pad Prism software version 7 was

used to perform statistical analysis. Mann-

Whitney test was applied in all

experiments. 


\section{Acknowledgements}

O.V. and L.J.C. were supported by project grants from the European Commission H2020-MSCA-RISE (644373 - PRISAR), H2020-MSCA-RISE (777682 - CANCER), H2020-WIDESPREAD-05-2017-Twinning (807281 - ACORN), H2020WIDESPREAD-2018-03 (852985 SIMICA), H2020-SCA-RISE-2016 (734684 - CHARMED) and MSCA-ITN2015-ETN (675743-ISPIC), 861190 (PAVE), 857894 (CAST), 859908 (NOVAMRI); 860173 (RISE-WELL); 872860 (PRISAR2). L.J.C was also supported by the research program VIDI (project number 723.012.110) of Dutch Research Council (NWO). C.E. was supported by the research program VENI with project number 916.181.54, which is (partly) financed by the Dutch Research Council (NWO) and the European Union's Horizon 2020 research and innovation programme under the Marie Sklodowska Curie grant agreement SIMICA (852985).

\section{Abbreviations}

Dichloromethane, DCM; dynamic light scattering, DLS; dimethylformamide, DMF; 9,10-diphenylanthracene, DPA; encapsulation efficiency, EE; intersystem crossing, ISC; nanoparticles, NPs; polydispersity index, PDI; poly(lactic-co- glycolic acid, PLGA; Platinum(II) octaethylporphyrin, PtOEP; TTA, triplettriplet annihilation; triplet-triplet energy transfer, TTET; upconversion, UC. 


\section{References}

Kinch, M. S. \& Woodard, P. in Drug

Discov Today (2017 July).

2 Chen, G., Qiu, H., Prasad, P. N. \&

Chen, $X$. Upconversion nanoparticles:

design, nanochemistry, and

applications in theranostics. Chem Rev

114, 5161-5214,

doi:10.1021/cr400425h (2014).

3 Zuo, J. et al. Near Infrared Light

Sensitive Ultraviolet-Blue

Nanophotoswitch for Imaging-Guided

"Off-On" Therapy. ACS Nano 12, 3217-

3225, doi:10.1021/acsnano.7b07393

(2018).

$4 \quad$ Nguyen, P. D., Son, S. J. \& Min, J.

Upconversion nanoparticles in

bioassays, optical imaging and

therapy. Journal of nanoscience and

nanotechnology 14, 157-174, doi:10.1166/jnn.2014.8894 (2014).

5

Singh, R., Dumlupinar, G., Andersson-

Engels, S. \& Melgar, S. Emerging

applications of upconverting

nanoparticles in intestinal infection

and colorectal cancer. Int $J$

Nanomedicine 14, 1027-1038, doi:10.2147/IJN.S188887 (2019).

Rauch, M. P. \& Knowles, R. R.

Applications and Prospects for Triplet-

Triplet Annihilation Photon

Upconversion. Chimia (Aarau) 72,

501-507,

doi:10.2533/chimia.2018.501 (2018).

7 Huang, L. et al. Designing next generation of photon upconversion:

Recent advances in organic triplet-

triplet annihilation upconversion

nanoparticles. Biomaterials 201, 77-

86 ,

doi:10.1016/j.biomaterials.2019.02.00

8 (2019).

8 Zhou, J., Liu, Q., Feng, W., Sun, Y. \& Li,

F. Upconversion luminescent

materials: advances and applications.

Chem Rev 115, 395-465,

doi:10.1021/cr400478f (2015).

9 Zhu, X., Su, Q., Feng, W. \& Li, F. Anti-

Stokes shift luminescent materials for bio-applications. Chem Soc Rev 46,
1025-1039, doi:10.1039/c6cs00415f

(2017).

10 Borisov, S. M., Saf, R., Fischer, R. \&

Klimant, I. Synthesis and properties of new phosphorescent red light-

excitable platinum(II) and

palladium(II) complexes with Schiff

bases for oxygen sensing and triplet-

triplet annihilation-based

upconversion. Inorg Chem 52, 1206-

1216, doi:10.1021/ic301440k (2013).

11 Han, J., Duan, P., Li, X. \& Liu, M.

Amplification of Circularly Polarized

Luminescence through Triplet-Triplet

Annihilation-Based Photon

Upconversion. J Am Chem Soc 139,

9783-9786, doi:10.1021/jacs.7b04611

(2017).

12 Sguerra, F., Bulach, V. \& Hosseini, M.

W. Molecular tectonics: zinc

coordination networks based on

centric and acentric porphyrins

bearing pyridyl units. Dalton Trans 41, 14683-14689,

doi:10.1039/c2dt32051g (2012).

13 Yang, Z.-S., Ning, Y., Yin, H.-Y. \&

Zhang, J.-L. Lutetium(iii) porphyrinoids as effective triplet photosensitizers for photon upconversion based on triplet-triplet annihilation (TTA). Inorganic Chemistry Frontiers 5, 22912299, doi:10.1039/C8QI00477C (2018).

14 Cruz, L. J. et al. Targeting nanosystems to human DCs via Fc receptor as an effective strategy to deliver antigen for immunotherapy. Mol Pharm 8, 104-116, doi:10.1021/mp100178k (2011).

15 Cruz, L. J. et al. Targeting nanoparticles to dendritic cells for immunotherapy. Methods Enzymol 509, 143-163, doi:10.1016/B978-0-12391858-1.00008-3 (2012).

16 Aarntzen, E. H. et al. Targeting of 111 In-labeled dendritic cell human vaccines improved by reducing number of cells. Clin Cancer Res 19, 1525-1533, doi:10.1158/10780432.CCR-12-1879 (2013). 
17 Ghinnagow, R. et al. Co-delivery of the NKT agonist alpha-galactosylceramide and tumor antigens to cross-priming dendritic cells breaks tolerance to self-antigens and promotes antitumor responses. Oncoimmunology 6 , e1339855, doi:10.1080/2162402X.2017.1339855 (2017).

18 Rueda, F. et al. Effect of TLR ligands co-encapsulated with multiepitopic antigen in nanoliposomes targeted to human DCs via Fc receptor for cancer vaccines. Immunobiology 222, 989997, doi:10.1016/j.imbio.2017.06.002 (2017).

19 Da Silva, C. G. et al. Co-delivery of immunomodulators in biodegradable nanoparticles improves therapeutic efficacy of cancer vaccines.

Biomaterials 220, 119417, doi:10.1016/j.biomaterials.2019.1194 17 (2019).

20 Da Silva, C. G. et al. Effective chemoimmunotherapy by co-delivery of doxorubicin and immune adjuvants in biodegradable nanoparticles. Theranostics 9, 6485-6500, doi:10.7150/thno.34429 (2019). Cruz, L. J. et al. Tracking targeted bimodal nanovaccines: immune responses and routing in cells, tissue, and whole organism. Mol Pharm 11, 4299-4313, doi:10.1021/mp400717r (2014).

22 Cruz, L. J. et al. Controlled release of antigen and Toll-like receptor ligands from PLGA nanoparticles enhances immunogenicity. Nanomedicine (Lond) 12, 491-510, doi:10.2217/nnm2016-0295 (2017).

23 Duan, P., Yanai, N. \& Kimizuka, N. Photon upconverting liquids: matrixfree molecular upconversion systems functioning in air. J Am Chem Soc 135, 19056-19059, doi:10.1021/ja411316s (2013).

24 Duan, P., Yanai, N., Nagatomi, H. \& Kimizuka, N. Photon upconversion in supramolecular gel matrixes: spontaneous accumulation of light- harvesting donor-acceptor arrays in nanofibers and acquired air stability. $J$ Am Chem Soc 137, 1887-1894, doi:10.1021/ja511061h (2015). Cruz, L. J., Tacken, P. J., Fokkink, R. \& Figdor, C. G. The influence of PEG chain length and targeting moiety on antibody-mediated delivery of nanoparticle vaccines to human dendritic cells. Biomaterials 32, 67916803 , doi:10.1016/j.biomaterials.2011.04.08 2 (2011).

26 Dzebo, D., Moth-Poulsen, K. \& Albinsson, B. Robust triplet-triplet annihilation photon upconversion by efficient oxygen scavenging. Photochem Photobiol Sci 16, 13271334, doi:10.1039/c7pp00201g (2017).

27 Bansal, A. K., Holzer, W., Penzkofer, A. \& Tsuboi, T. Absorption and emission spectroscopic characterization of platinum-octaethyl-porphyrin (PtOEP). Chemical Physics 330, 118129, doi:https://doi.org/10.1016/j.chemph ys.2006.08.002 (2006).

28 Zuckerman, J. E., Choi, C. H., Han, H. \& Davis, M. E. Polycation-siRNA nanoparticles can disassemble at the kidney glomerular basement membrane. Proc Natl Acad Sci U S A 109, 3137-3142, doi:10.1073/pnas.1200718109 (2012).

29 Bae, Y. H. \& Park, K. Targeted drug delivery to tumors: myths, reality and possibility. J Control Release 153, 198205, doi:10.1016/j.jconrel.2011.06.001 (2011).

30 S Honary, F. Z. Effect of Zeta Potential on the Properties of Nano-Drug

Delivery Systems - A Review (Part 2). Tropical Journal of Pharmaceutical Research doi:https://www.ajol.info/index.php/t ipr/article/view/88556 (2013).

31 Company, A. L. Physical Stability of Disperse Systems. Vol 1 (2009).

$32 \mathrm{Li}, \mathrm{X}$. et al. Dependence between cytotoxicity and dynamic subcellular 
localization of up-conversion nanoparticles with different surface charges. RSC Advances 7, 3350233509, doi:10.1039/C7RA04487A (2017).

33 M Haase, H. S. Upconverting Nanoparticles. Angew Chem Int Ed Engl., doi:10.1002/anie.201005159 (2011).

34 Schmidt, T. W. \& Castellano, F. N. Photochemical Upconversion: The Primacy of Kinetics. J Phys Chem Lett 5, 4062-4072, doi:10.1021/jz501799m (2014).

35 Ogawa, T., Yanai, N., Monguzzi, A. \& Kimizuka, N. Highly Efficient Photon Upconversion in Self-Assembled LightHarvesting Molecular Systems. Sci Rep 5, 10882, doi:10.1038/srep10882 (2015).

36 Makadia, H. K. \& Siegel, S. J. Poly Lactic-co-Glycolic Acid (PLGA) as Biodegradable Controlled Drug Delivery Carrier. Polymers (Basel) 3, 1377-1397, doi:10.3390/polym3031377 (2011).

37 Kalyane, D. et al. Employment of enhanced permeability and retention effect (EPR): Nanoparticle-based precision tools for targeting of therapeutic and diagnostic agent in cancer. Materials science \& engineering. $C$, Materials for biological applications 98, 1252-1276, doi:10.1016/j.msec.2019.01.066 (2019).

38 Hashizume, H. et al. Openings between defective endothelial cells explain tumor vessel leakiness. Am J Pathol 156, 1363-1380, doi:10.1016/S0002-9440(10)65006-7 (2000). 